Check for updates

Cite this: J. Mater. Chem. B, 2020, 8, 9162

DOI: $10.1039 / d 0 t b 90169 e$

rsc.li/materials-b

\section{Correction: Enabling nanopore technology for sensing individual amino acids by a derivatization strategy}

\author{
Xiaojun Wei, ${ }^{\text {ab }}$ Dumei Ma, ${ }^{\text {cd }}$ Lihong Jing, ${ }^{\text {e }}$ Leon Y. Wang, ${ }^{\mathrm{b}}$ Xiaoqin Wang, ${ }^{\mathrm{b}}$ \\ Zehui Zhang, ${ }^{a}$ Brian J. Lenhart, ${ }^{b}$ Yingwu Yin, ${ }^{d}$ Qian Wang*c and Chang Liu*ab
}

Correction for 'Enabling nanopore technology for sensing individual amino acids by a derivatization strategy' by Xiaojun Wei et al., J. Mater. Chem. B, 2020, 8, 6792-6797, DOI: 10.1039/D0TB00895H.

An additional citation of a related ACS Sensors paper ${ }^{1}$ from the authors entitled 'N-Terminal Derivatization-Assisted Identification of Individual Amino Acids Using a Biological Nanopore Sensor' should have been included as ref. 52 in the final paragraph on page 6792. The first few sentences of this paragraph should read as follows:

'Based on the Edman peptide degradation reaction, ${ }^{9,37-39}$ a proof-of-concept of derivatization assisted identification of amino acids by nanopore has been demonstrated. ${ }^{52}$ Herein we further demonstrate that the efficient $\mathrm{N}$-terminal derivatization of amino acids using aromatic tags can augment the distinguishability of different amino acids with similar properties when they translocate through $\alpha$-hemolysin $(\alpha$-HL) nanopores.'

Instead of:

'Based on the Edman peptide degradation reaction, ${ }^{9,37-39}$ herein we demonstrate that the efficient N-terminal derivatization of amino acids using aromatic tags can augment the distinguishability of different amino acids when they translocate through $\alpha$-hemolysin $(\alpha-\mathrm{HL})$ nanopores.'

The authors also wish to clarify the differences between this Journal of Materials Chemistry B paper and the ACS Sensors paper. The ACS Sensors paper demonstrates that N-terminal derivatisation strategies can assist the differentiation of amino acids with an emphasis on the impact of N-terminal derivatisation on the translocation of amino acids crossing nanopores. The Journal of Materials Chemistry $B$ paper focuses on the differentiation of three groups of similar amino acids using NITC and NDA derivatisation.

The Royal Society of Chemistry apologises for these errors and any consequent inconvenience to authors and readers.

\title{
References
}

1 X. Wei, D. Ma, Z. Zhang, L. Y. Wang, J. L. Gray, L. Zhang, T. Zhu, X. Wang, B. J. Lenhart, Y. Yin, Q. Wang and C. Liu, ACS Sens., 2020, 5, 1707-1716.

\footnotetext{
${ }^{a}$ Biomedical Engineering Program, University of South Carolina, Columbia, SC 29208, USA. E-mail: changliu@cec.sc.edu

${ }^{b}$ Department of Chemical Engineering, University of South Carolina, Columbia, SC 29208, USA

${ }^{c}$ Department of Chemistry and Biochemistry, University of South Carolina, Columbia, SC 29208, USA. E-mail: wang263@mailbox.sc.edu

${ }^{d}$ Department of Chemical and Biochemical Engineering, College of Chemistry and Chemical Engineering, Xiamen University, Xiamen 361005, Fujian, China

${ }^{e}$ Key Laboratory of Colloid, Interface and Chemical Thermodynamics, Institute of Chemistry, Chinese Academy of Sciences, Bei Yi Jie 2, Zhong Guan Cun, Beijing 100190,

China. E-mail: jinglh@iccas.ac.cn
} 